\title{
NAG1-1779
}

AIAA-97-1866

\section{A MULTIBLOCK APPROACH FOR CALCULATING INCOMPRESSIBLE FLUID FLOWS ON UNSTRUCTURED GRIDS}

\author{
Chunhua Sheng $^{*}$ and David L. Whitfield ${ }^{\dagger}$ \\ Computational Fluid Dynamics Laboratory \\ NSF Engineering Research Center, Mississippi State, MS 39762 \\ W. Kyle Anderson $\ddagger$ \\ NASA Langley Research Center, Hampton, VA 23681
}

\begin{abstract}
A multiblock approach is presented for solving two-dimensional incompressible turbulent flows on unstructured grids. The artificial compressibility form of the governing equations is solved by a vertex-centered, finite-volume implicit scheme which uses a backward Euler time discretization. Point Gauss-Seidel relaxations are used to solve the linear system of equations at each time step. This work introduces a multiblock strategy to the solution procedure, which greatly improves the efficiency of the algorithm by significantly reducing the memory requirements while not increasing the CPU time. Results presented in this work shows that the current multiblock algorithm requires $70 \%$ less memory than the single block algorithm.
\end{abstract}

\section{Introduction}

In the past decade, much progress has been made in developing computational techniques for predicting flow fields about complex configurations. These techniques include both structured and unstructured grid algorithms, which have their own advantages and disadvantages. The biggest advantage of the unstructured grid approach over the structured grid approach is that the process of grid generation for complex geometries is greatly simplified. Another advantage is that unstructured grids lend themselves to adaptive grid methods because new nodes can be added to a localized region of the mesh by modifying a small subset of the overall grid data structure. Although the unstructured grid approach enjoys these advantages over structured grids, flow solvers that utilize it suffer several disadvantages. These primarily include a factor of 2-3 increase in memory requirements and computer run times on a per grid point basis [1].

\footnotetext{
${ }^{*}$ Research Engineer, Member AIAA

${ }^{\dagger}$ Professor, Member AIAA

${ }^{\ddagger}$ Senior Research Scientist, Member AIAA

Copyright $(1997$ by the American Institute of Aeronautics and Astronautics, Inc. All rights reserved.
}

This work introduces a multiblock approach to calculate the two-dimensional incompressible Euler and Navier-Stokes flows based on unstructured grids. The multiblock technique has been widely and successfully used in solution algorithms of structured grids to solve flows about complex configurations [2][3]. The application of the multiblock approach in the unstructured grid area seems not to have received much attention. Part of the reason for that is due to the fact that computational meshes for complex geometries can be relatively easily generated using unstructured grids without the aid of the multiblock technique. However, studies of structured grid algorithms by the present authors found that the multiblock approach can also significantly reduce the memory requirements by almost one order of magnitude in real-world flow computations [2][3]. These studies and findings motivated the current work to use the same multiblock concept to reduce the memory requirements of an unstructured grid algorithm.

The baseline code was originally developed at NASA Langley Research Center called FUN2DI [1]. It solves the artificial compressibility form of the two-dimensional incompressible Euler and Navier Stokes equations on unstructured meshes. Two different turbulence models (Baldwin and Barth [4] and Spalart and Allmaras [5]) are included in the code for turbulent flow computations, although the current work only uses the latter model. The discretized scheme uses a vertex-centered finite-volume upwind approximation with edgebased data structure. The numerical fluxes for the inviscid part are evaluated using the flux-difference splitting of Roe scheme. The viscous terms are evaluated with a finite-volume formulation that is equivalent to a Galerkin type of approximation [6]. The time-advancement algorithm is based on the linearized backward Euler time-difference scheme, which yields a linear system of equations for the solution at each time step. The Gauss-Seidel procedure is used to solve the linear system of equations at each time step. The original version of the code also has the option to apply FMG-FAS multigrid $\mathrm{V}$ or $\mathrm{W}$ cycles to accelerate the convergence of the solution to a steady state. 
The idea to use the multiblock approach to reduce the memory requirements of the algorithm is quite simple. Instead of solving the equations for all the nodes of the grid at one time, the solution process is broken down into several pieces (blocks) on the grid, which is performed one by one. Because only one block is processed at any time, the memory is allocated to store the data for that block, which requires much less memory than that to store the data for the whole grid. Special attention should be paid to the block interfaces where the information must be properly passed from the other blocks.

This paper is organized as follows. The artificial compressibility form of the two-dimensional $\mathbf{R e}$ ynolds-averaging Navier-Stokes equations is first outlined, followed by the numerical procedures used in Ref. [1]. The multiblock algorithm is introduced next, which mainly includes grid decomposition, multiblock implementation, and boundary treatment at block interfaces. Solutions of turbulent flows about a NACA 4412 airfoil and two 4-element airfoils are presented to demonstrate the efficiency and accuracy of the current multiblock algorithm. Some conclusions are summarized in the last section.

\section{Governing Equations}

Incompressible Navier-Stokes Equations

The unsteady two-dimensional incompressible Reynolds-averaged Navier-Stokes equations without body forces are written in Cartesian coordinates and in conservative form. A pseudo-time derivative of pressure is added to the continuity equation. The resulting set of equations in integral form represents a system of conservation laws for a control volume that relates the rate of change of a vector of average state variables $q$ to the flux through the volume surface, which can be written as

$$
V \frac{\partial q}{\partial t}+\oint_{\partial \Omega} \overrightarrow{f_{i}} \cdot \hat{n} d l-\oint_{\partial \Omega} \overrightarrow{f_{\nu}} \cdot \hat{n} d l=0
$$

where $\hat{n}$ is the outward-pointing unit normal to the control volume $V$. The vector of dependent state variables $q$ and the inviscid and viscous fluxes normal to the control volume $\vec{f}_{i}$ and $\vec{f}_{v}$ are given as

$$
\begin{gathered}
q=\left[\begin{array}{l}
p \\
u \\
v
\end{array}\right] \\
\overrightarrow{f_{i}} \cdot \hat{n}=\left[\begin{array}{c}
\beta \Theta \\
u \Theta+n_{x} p \\
v \Theta+n_{y} p
\end{array}\right]
\end{gathered}
$$

$$
\overrightarrow{f_{v}} \cdot \hat{n}=\left[\begin{array}{c}
0 \\
n_{x} \tau_{x x}+n_{y} \tau_{x y} \\
n_{x} \tau_{x y}+n_{y} \tau_{y y}
\end{array}\right]
$$

where $\beta$ is the artificial compressibility parameter; $u$ and $v$ are the Cartesian velocity components in the $x$ and $y$ directions, respectively; $\Theta$ is the velocity normal to the surface of the control volume, where

$$
\Theta=n_{x} u+n_{y} v
$$

and $p$ is the pressure. Note that the variables in the above equations are nondimensionalized with the characteristic length, freestream values of velocity, density, and viscosity. Pressure is normalized using the following relationship $\left(p-p_{\infty}\right) / \varrho_{\infty} V_{\infty}^{2}$, where the subscript denotes a freestream or reference value. The shear stresses in $\mathrm{Eq}$. (1) are given as

$$
\begin{aligned}
& \tau_{x x}=\left(\mu+\mu_{t}\right) \frac{2}{\operatorname{Re}} u_{x} \\
& \tau_{y y}=\left(\mu+\mu_{i}\right) \frac{2}{\operatorname{Re}} v_{y} \\
& \tau_{x y}=\left(\mu+\mu_{t}\right) \frac{1}{\operatorname{Re}}\left(u_{y}+v_{x}\right)
\end{aligned}
$$

where $\mu$ and $\mu_{r}$ are the laminar and turbulent viscosities, respectively, and $R e$ is the Reynolds number.

\section{Solution Algorithm}

\section{Finite-Volume Scheme}

The baseline flow solver is a node-centered finitevolume implicit scheme. The computational domain is divided into a finite number of triangles from which control volumes are formed that surround each vertex in the mesh. The flow variables are stored at the vertices of the triangle. Equation (1) is then numerically integrated over the closed boundaries of the control volumes surrounding each node. These control volumes are formed by connecting the center of each triangle to the midpoint of the edges, as shown in Figure 1. These nonoverlapping control volumes combine to completely cover the domain and are considered to form a mesh which is dual to the mesh composed of triangles formed from the vertices.

\section{Numerical Flux Evaluation}

The numerical evaluation of the surface integrals in Eq. (1) is conducted separately for the inviscid and viscous contributions. The inviscid fluxes are obtained on the faces of each control volume with a flux-difference-splitting scheme, while the viscous terms are evaluated with a finite-volume formulation that is equivalent to a Galerkin type of approximation [1]. The inviscid fluxes on the boundaries of the control volumes are given by 


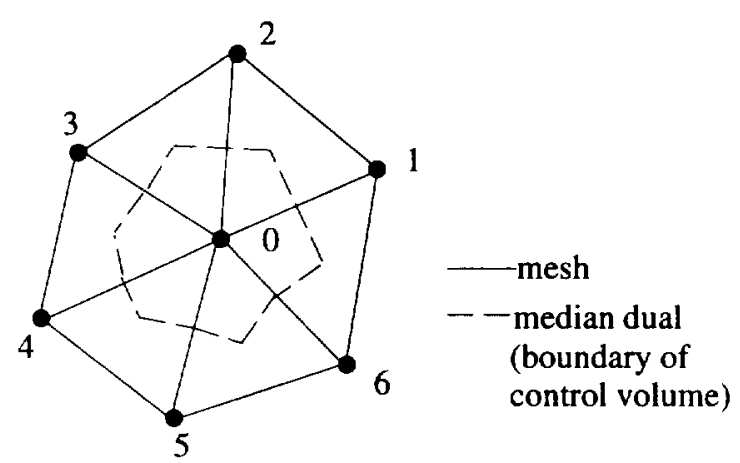

Figure 1. Control volume surrounding a node

$$
\begin{aligned}
\Phi=\frac{1}{2}\left(f_{i}\left(q^{+} ; \hat{n}\right)\right. & \left.+f_{i}\left(q^{-} ; \hat{n}\right)\right) \\
& -\frac{1}{2}|\bar{A}|\left(q^{+}-q^{-}\right)
\end{aligned}
$$

where $\Phi$ is the numerical flux, and $f_{i}$ is the flux vector given in Eq. (1). A nonsingular eigensystem for the matrix $|\bar{A}|$ was obtained in Ref. [1] and will not be repeated here. Quantities $q^{+}$and $q^{-}$are the values of the dependent variables on the left and right side of the boundary of the control volume. For first-order accurate differencing, the data on the left and right sides of the cell face ( $q^{+}$and $q^{-}$) are set equal to the data at the nodes lying on either side of the cell face. For higher-order differencing, these values are computed with a Taylor series expansion about the central node of the control volume

$$
q_{\text {face }}=q_{\text {node }}+\nabla q \cdot \vec{r}
$$

where $\vec{r}$ is the vector that extends from the central node to the midpoint of each edge, and $\nabla q$ is the gradient of the dependent variables at the node.

The gradient $\nabla q$ can be evaluated with a leastsquares procedure in which the data surrounding each node is assumed to behave linearly. The data at each node surrounding the center node may be expressed as

$$
\begin{aligned}
q_{i}=q_{o} & +q_{x_{o}}\left(x_{i}-x_{o}\right) \\
& +q_{y_{o}}\left(y_{i}-y_{o}\right)
\end{aligned}
$$

By expressing the data in a like manner at each of the $N$ surrounding nodes, an $N \times 2$ system of equations is formed as

$$
\left[\begin{array}{cc}
\Delta x_{1} & \Delta y_{l} \\
\Delta x_{2} & \Delta y_{2} \\
\vdots & \vdots \\
\Delta x_{N} & \Delta y_{N}
\end{array}\right] \quad\left\{\begin{array}{l}
q_{x_{o}} \\
q_{y_{0}}
\end{array}\right\}=\left[\begin{array}{cc}
q_{1}-q_{0} \\
q_{2}-q_{0} \\
\vdots & \vdots \\
q_{N}-q_{0}
\end{array}\right]
$$

The equation above represents an overdetermined system, which can be solved by using the normal equation approach [7] to obtain the gradients at the nodes. Anoth- er approach to calculate the gradients at the nodes, as suggested in Ref. [6], is to use the Gram-Schmidt process because of the sensitivity of the normal equation approach to the condition number squared of the solution matrix [6]. The resulting formulas for calculating the gradients at the center node in Figure 1 are given as

$$
\begin{aligned}
& q_{x_{o}}=\sum_{i=1}^{N} W_{i}^{x}\left(q_{i}-q_{o}\right) \\
& q_{y_{0}}=\sum_{i=1}^{N} W_{i}^{y}\left(q_{i}-q_{o}\right)
\end{aligned}
$$

where the summation is over all the edges that connect to the node and the weights are given by

$$
\begin{aligned}
& W_{i}^{x}=\frac{\left[r_{y y}\left(x_{i}-x_{o}\right)-r_{x y}\left(y_{i}-y_{0}\right)\right]}{r_{x x} r_{y y}-r_{x y}^{2}} \\
& W_{i}^{y}=\frac{\left[r_{x x}\left(y_{i}-y_{o}\right)-r_{x y}\left(x_{i}-x_{0}\right)\right]}{r_{x x} r_{y y}-r_{y y}^{2}}
\end{aligned}
$$

with

$$
\begin{gathered}
r_{x x}=\sum_{i=1}^{N}\left(x_{i}-x_{o}\right)^{2}, \quad r_{y y}=\sum_{i=1}^{N}\left(y_{i}-y_{o}\right)^{2} \\
r_{x y}=\sum_{i=1}^{N}\left(x_{i}-x_{o}\right)\left(y_{i}-y_{o}\right)
\end{gathered}
$$

Notice that the above Eq. (5) gives unweighted gradients in which all the data surrounding the central node are given equal consideration. It was found in Ref. [6] that weighted gradients, which are evaluated by using inverse distance or Green's theorem, provide better accuracy than unweighted gradients when actual gradients are required, as in the production terms for the turbulence model. However, for reconstructing nonlinear data on highly stretched meshes, such as viscous grids, an unweighted formulation is far superior to either inverse distance weighting or the use of gradients calculated with Green's theorem [6].

The viscous flux contribution to the residual is obtained using a finite-volume approach. In this approach, quantities such as velocity derivatives are first evaluated in each triangle of the mesh and the viscosity is computed as an average of the three nodes making up the triangle.

\section{Time Advancement Scheme}

The time-advancement algorithm is based on the linearized backward Euler time-differencing scheme, which yields a linear system of equations for the solution at each time step:

$$
[A]^{n} \quad\{\Delta q\}^{n}=\{r\}^{n}
$$

where $\{r\}^{n}$ is the vector of steady-state residuals, $\{\Delta q\}^{n}$ represents the change in the dependent variables, and the solution matrix $[A]^{n}$ is written as 


$$
[A]^{n}=\frac{V}{\Delta t} I+\frac{\partial r}{\partial q}
$$

The solution of this system of equations is obtained by a relaxation scheme in which $\{\Delta q\}^{n}$ is obtained through a sequence of iterations, $\{\Delta q\}^{i}$, which converge to $\{\Delta q\}^{n}$. There are several variations of classic relaxation procedures which have been used in the past for solving this linear system of equations [8][9]. In this work, a point implicit Gauss-Seidel procedure as described in Ref. [1] is used. To clarify the scheme, $[\mathrm{A}]^{n}$ is first written as a linear combination of two matrices representing the diagonal and off-diagonal terms:

$$
[A]^{n}=[D]^{n}+[O]^{n}
$$

and the solution to the linear system of equations is obtained by adopting a Gauss-Seidel type of strategy in which all odd-numbered nodes are updated first, followed by the solution of the even-numbered nodes. This procedure can be represented as

$$
[D]^{n}\{\Delta q\}^{i+1}=\left[\{r\}^{n}-[O]\{\Delta q\}^{(i+1) / i}\right]
$$

where $\{\Delta q\}^{(i+1) / i}$ is the most recent value of $\Delta q$, which will be at subiteration level $i+1$ for the odd-numbered nodes that have been previously updated and at level $i$ for the even-numbered nodes. Normally 15-20 subiterations are adequate at each time step.

\section{Turbulence Modelling}

For the current study, the one-equation turbulence model of Spalart and Allmaras is used [5]. Attention is paid to the distance function when coupling this turbulence model with the multiblock algorithm described next. That is, the distance function for each node of the grid is computed based on the closest distance to the wall across all blocks, prior to processing each block. In the solution process, the equation for turbulent viscosity is solved using a backward-Euler time-stepping scheme similar to that used for the flow variables, but separated from the flow equations, This results in a loosely coupled solution process that allows easy interchange with new turbulence models. For more details about the turbulent model and its implementation, see Refs. [5] and [1].

\section{Multiblock Algorithm}

\section{Grid Decomposition}

In the process to generate a structured grid in multiblocks, the physical domain is first divided into several subdomains with prescribed block boundaries. The grid in each block is then generated separately. To generate a multiblock unstructured grid, however, the above pro- cess does not apply because prescribed block boundaries will degrade the grid quality in these regions. The multiblock unstructured grid is generated through a grid decomposition process. First, the computational grid of the whole physical domain is constructed within a single block using a grid generation program called TRI8IT [1]. Then, a domain decomposition method is used to break the mesh into several sub-domains (or blocks) by selecting all the cells that fall into the region set for each subdomain. Each block contains a complete set of node information, just like a separate grid. A file which contains information to connect block-to-block interfaces is also created. It was found that this domain decomposition method has also been used for other purposes in unstructured grid algorithms, such as for parallel computations [10].

There are several criteria that are used to guide the process of the grid decomposition. The first one is the number of nodes or cells in each block. Since the overall memory requirements of the code are determined by the largest sized block, each block grid should contain a nearly equal number of grid nodes or cells to achieve the best reduction in memory usage. The second criterion is the smoothness of block interfaces. A sawtoothed block boundary, which often occurs near the wall surfaces in viscous grids, should be avoided because the accuracy of gradients of flow variables along that boundary can be severely compromised. Other criterion such as selecting the least number of interfaces for a given number of blocks to minimize the data communication between blocks, should also be considered. Unfortunately, these criteria are not automatically implemented in the current version of the grid decomposition code, but only through careful selection and adjustment of the values that define the region for each block.

\section{Multiblock Strategy and Memory Allocation}

The implementation of the multiblock algorithm on unstructured grids adopts a similar strategy as used in structured grids in Ref. [2], i.e. a "vertical mode" in which a complete cycle is completed in each block before proceeding to the next block. The advantage of this approach is that the solution process (nonlinear and linear procedure) in each block is local and thus does not depend on the solution in other blocks. The data at block interfaces are treated as block boundary conditions which are updated after each time step, as described below. This nature of independence of the solution to other blocks offers great flexibility in both implementation and memory allocation for the algorithm, and also provides a natural platform for parallel implementations. (In fact, the main difference between the multiblock algorithm and the parallel implementation is that in the former, the solution in each block is performed sequentially in a prescribed order, while in the latter, all blocks 
are solved simultaneously). The disadvantage of the "vertical mode" is that if a grid has a lot of blocks, the convergence rate could suffer because of the explicit nature of the data interchange between blocks. Since the main purpose of this work is to reduce the memory requirements of the unstructured grid algorithm, the memory allocation in the code must be done in a special way to achieve the best efficiency in both memory usage and CPU time. In the current work, all memory is allocated either locally or globally. For local memory, only the storage needed for the current block is allocated when the solution process enters that block, and this storage is freed at the time when the solution process leaves the block. The new storage is allocated again when the solution process moves to the next block. The local memory allocation is mainly for variables which do not need to be stored for all blocks, such as the flux Jacobian matrix (most costly part in the memory usage) which is updated after each time step in each block. On the other hand, the global memory allocation means that storage is allocated for all blocks and is not freed until the end of the iterations. Some data such as conserved flow variables, grid coordinates and the distance function in the turbulence model, must be stored in a global way for all blocks. Therefore, by adopting the above strategy for memory allocation, the memory requirements of the multiblock algorithm will be much less than that of the single block algorithm where all memory is allocated globally.

Notice that the way to compute and save the metric quantities of the grid has great impact on the overall memory usage in the multiblock algorithm, because the storage for these metric terms is the second largest part in the memory cost. If the metric terms of the grid are computed and saved in each block, the memory reduction by this method is limited to the maximum of $45 \%$ of the single grid algorithm. The reason for that is because the memory needed to store these metric terms will exceed the memory reduced by the above multiblock algorithm when a certain number of blocks is exceeded. An improvement can be made by allocating these metric terms locally for each block and recomputing them after each time step. Although this will introduce some CPU time overhead, it is the most universal way for general unsteady problems with dynamic moving grids, because for these problems the metric terms of the grid must be recalculated after each time step following the grid movement anyway. In fact, since the memory usage of the algorithm is further reduced by this method, the overall CPU time to execute the code may be even less, because more data in the main memory can now reside in a much faster accessible data cache device which allows more efficient implementation of the code. Results in this work show that by adopting the above strategy, the memory savings by the multi- block algorithm is significantly increased to up to $70 \%$ of the single block algorithm, while the CPU time cost is even $12 \%$ less than that of the multiblock code in which the metric terms of the grid are allocated globally for all blocks, and $24 \%$ less than that of the original single block algorithm. In fact, over one order of magnitude in memory reduction is possible when the grid is divided into more blocks.

\section{block 1}

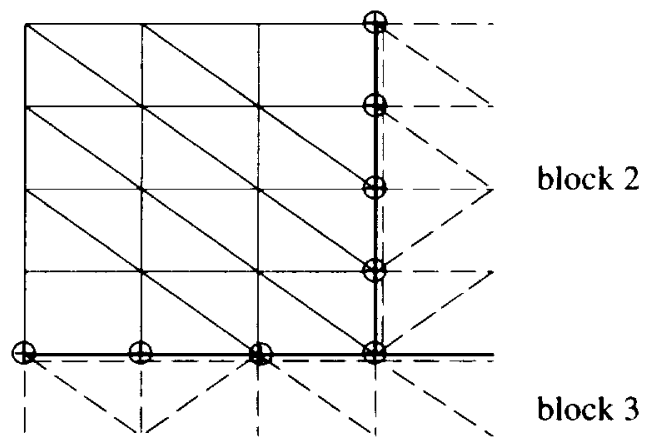

$\otimes \quad$ nodes at block interface

$\begin{array}{ll}- & \text { block interfaces } \quad \text { interior edges } \\ -- & \text { phantom edges stored in buffer arrays }\end{array}$

Figure 2. Nodes at block interfaces

\section{Interface Treatment}

Two major issues in the multiblock implementation are the data structure and the treatment of the block interfaces where two or more blocks are next to each other. A natural choice is to use the same edge-based data structure to store the data at block interfaces, and to treat the nodes at the interface in the same way as the interior nodes, see Figure 2 . In the algorithm, a set of buffer arrays is declared for each block to store the data on phantom edges that surround the current block and have the connection to the block interfaces (shown as dash lines in Figure 2). In the actual computation, the nodes at block interfaces (shown as circles in Figure 2) are computed in two steps. In the first step, contributions from interior edges (shown as solid lines in Figure 2) are collected and values are stored at the nodes. This procedure is the same as for all interior nodes. In the second step, contributions from other blocks are added to these nodes at block interfaces by looping over the phantom edges surrounding the current block using the data stored in the buffer arrays. The data in the buffer arrays are updated after each time step when all blocks have been processed. Notice that the above method provides synchronized boundary conditions at the block interfaces, since the gradient of flow variables is computed based on the values at the same previous time step. This ensures the performance of the multiblock algorithm to be 
close to that of the single block algorithm. It should be pointed out that an approximation is made here when calculating the flux contributions from the phantom edges. That is, the gradients at both nodes on each phantom edge are assumed to be the same, which take the value calculated at the node that is lying on the block interfaces. The reason for such an approximation is for the purpose of memory savings. However, numerical tests show that the accuracy of the multiblock algorithm is not significantly affected by this approximation, as discussed below.

\section{$\underline{\text { Results }}$}

Results are shown below for three airfoil cases, a NACA 4412 airfoil and two 4-element airfoils. The unstructured grids are first generated in a single block with a code described in Ref. [1], and a domain decomposition method is used to divide the grid into several blocks. Solutions are obtained with the single block and the multiblock algorithms, in which no multigrid scheme is used in both algorithms. Comparison is made for the memory usage and CPU time between the two methods. Notice that for both multiblock and single block algorithms, the metric quantities of the grid are recomputed after each time step. For each case, the CFL number has been linearly ramped from 20 to 200 over 100 iterations and 15 subiterations were used at each time step to obtain an approximate solution of the linear system. All computations were carried out on an SGI R $10000194 \mathrm{MHz}$ single processor with $2 \mathrm{~GB}$ in-core memory and $2 \mathrm{MB}$ data cache size.

\section{NACA 4412 Airfoil}

The first case selected to test the current algorithm is the viscous flow over a NACA 4412 airfoil. Computations are performed with both the single block and multiblock algorithms, and results are compared with the experimental data obtained in Ref. [11]. The flow conditions include an angle of attack of $13.87^{\circ}$, a Reynolds number of 1.52 million (based on the chord length of the airfoil). The unstructured grid contains 10347 nodes and 20694 cells. The normalized grid spacing at the wall is about $1 . \times 10^{-5}$. The multiblock grid is obtained by dividing the single block grid into three blocks, with the number of nodes of 3030,3625 , and 3864 in each block, respectively (Figure 3 ).

Figure 4 shows the convergence histories for both single block and multiblock algorithms. Both solutions have about the same convergence rate which indicates that the efficiency of the multiblock algorithm is not degraded with the current multiblock strategy and the treatment of block interfaces. Figure 5 shows the comparison of the $\mathrm{Cp}$ distributions on the airfoil surface between the experiment and computations. The result

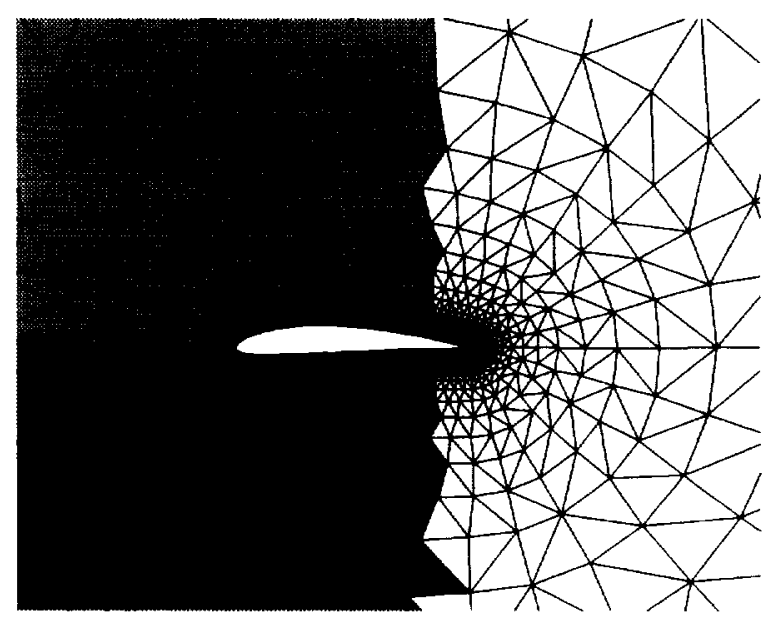

Figure 3. 3-block grid for NACA 4412 airfoil

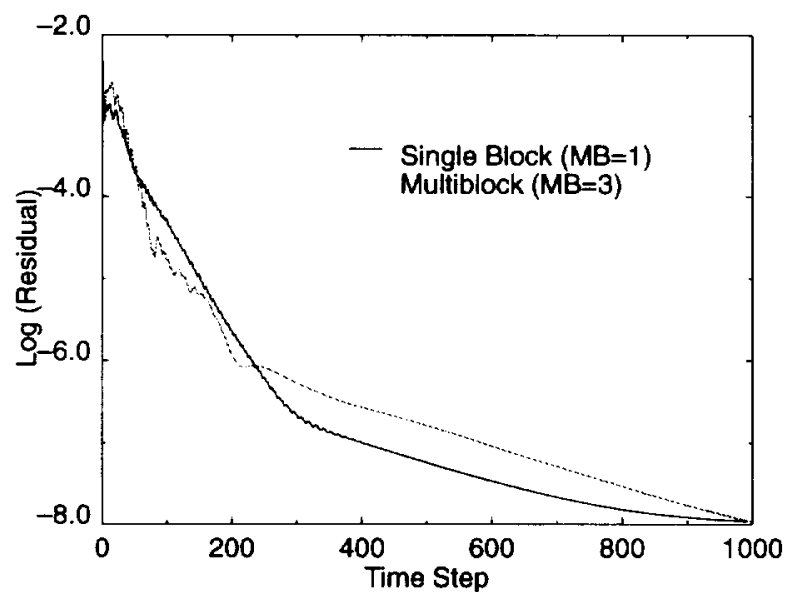

Figure 4. Convergence histories of single block and multiblock solutions

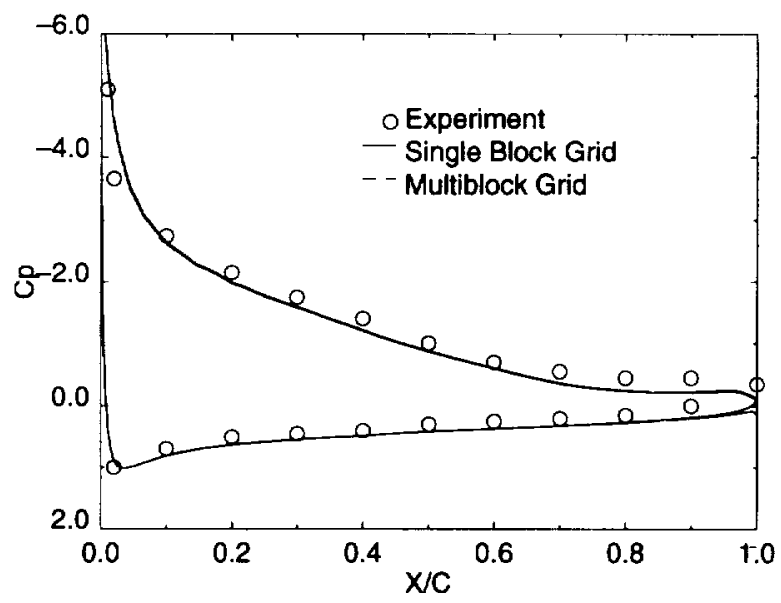

Figure 5. Comparison of $\mathrm{Cp}$ distributions between the experimental data and computations 


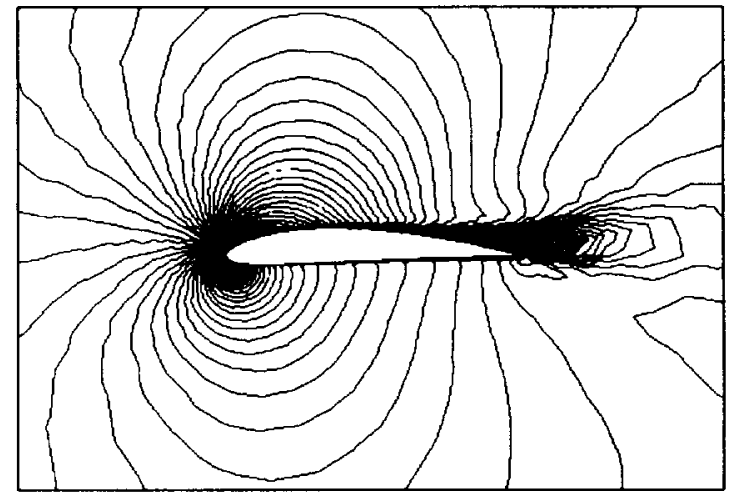

single block

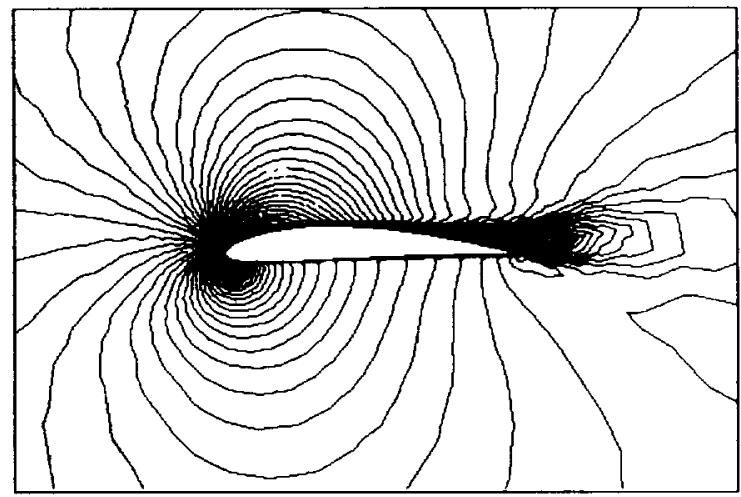

Multiblock

Figure 6. Computed u velocity components around the airfoil

shows that both multiblock and single block solutions are very close to each other, which are in good agreement with the experimental data. Figure 6 shows $u$ velocity contours of both the single block and multiblock solutions. The continuity of the contour lines across the interfaces indicates the proper treatment of passing data among blocks. The following Table 1 shows the memory usage and CPU times for 1000 time iterations for both single block and multiblock solutions. It is seen that the memory requirements for the 3-block multiblock code are $41 \%$ less than that for the single block grid.

\begin{tabular}{|l|l|l|c|}
\hline $\begin{array}{l}\text { Grid } \\
\text { (nodes) }\end{array}$ & Block & $\begin{array}{l}\text { Memory } \\
\text { (MB) }\end{array}$ & $\begin{array}{l}\text { CPU Time } \\
\text { (mins) }\end{array}$ \\
\hline 10347 & 1 & 22 & 42 \\
\hline 10347 & 3 & 13 & 38 \\
\hline
\end{tabular}

Table 1. Memory usage and CPU time of single block and multiblock solutions for NACA 4412 airfoil

\section{4-Element Airfoil}

To further demonstrate the efficiency of the current multiblock algorithm, computations were performed on two 4-element airfoils. The unstructured mesh for the first configuration was built with 34295 nodes and 68596 cells, which was decomposed into 8 blocks (Figure 7). It should be pointed that the block boundaries are placed in several critical wake regions to test the robustness and efficiency of the current code, as well as the accuracy of the block boundary condition as described before. The normalized grid spacing on the wall is $1 . \times 10^{-5}$, and the Reynolds number is 9 million, based on the length of the whole airfoil. Computations are performed for the flow at $0^{\circ}$ and $16^{\circ}$ angles of attack, and results are compared with the experimental data in Ref. [12].

Figure 8 and Figure 9 show the convergence histories of both multiblock and single block solutions at $0^{\circ}$ and $16^{\circ}$ angles of attack. The convergence rates of the multiblock and single block solutions are very similar for both flow conditions. The computed and measured Cp distributions on the slat, main, main flap, and auxiliary flap surfaces are shown in Figure 10 and Figure 11. Computed results are generally matched very well with the experimental data at both $0^{\circ}$ and $16^{\circ}$ angles of attack. However, a slight difference was found between the single block and multiblock solutions on the slat at $0^{\circ}$ angle of attack, which may be due to the approximation used for the gradients at the nodes on phantom edges or the sawtoothed block interfaces in the multiblock grid.

A numerical test was also conducted on another 4-element airfoil for which the unstructured mesh was built with 132331 nodes and 264668 cells, and was divided into 10 blocks (Figure 12). The normalized grid spacing on the wall is $1 . \times 10^{-6}$. The inflow conditions include $20.318^{\circ}$ angle of attack and a Reynolds number of 9 million. Figure 13 shows the contours of the $u$ velocity components obtained by both single block and multiblock solutions. It is seen that overall flow patterns between the two solutions are very similar. Figure 14 shows the convergence histories of both multiblock and single block solutions. It shows that the performance of the multiblock solution is not degraded when the number of blocks is increased.

Table 2 summarizes the CPU times for 1000 time steps and memory requirements for the above two grids. It is seen that the memory savings by the multiblock solution over the single block solution is $63 \%$ for the 8-block grid case, and $70 \%$ for the 10 -block grid case. The CPU time for the multiblock solutions is about $30-40 \%$ less than that required in the single block solu- 


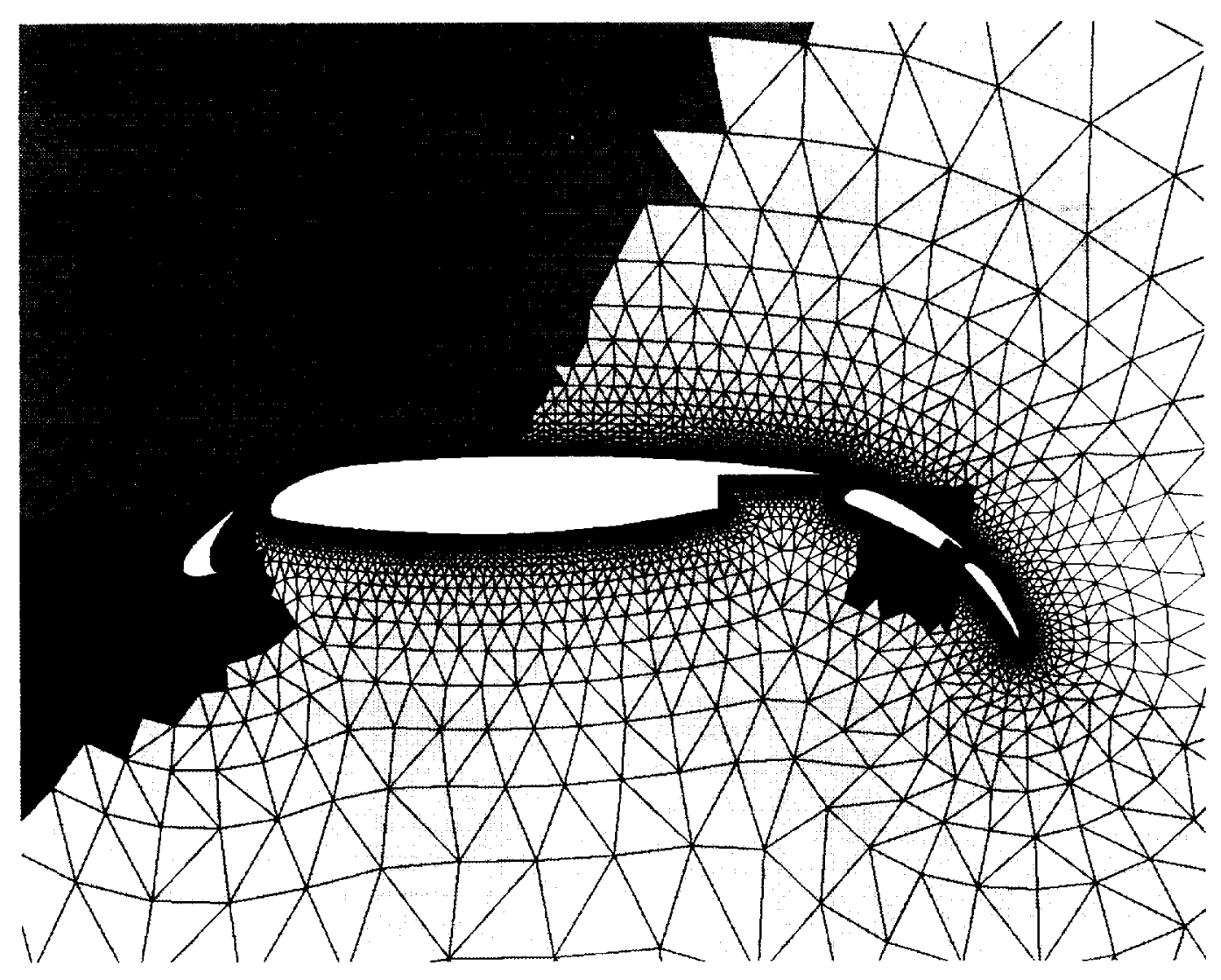

Figure 7. 8-block grid for the 4-element airfoil with 34295 nodes

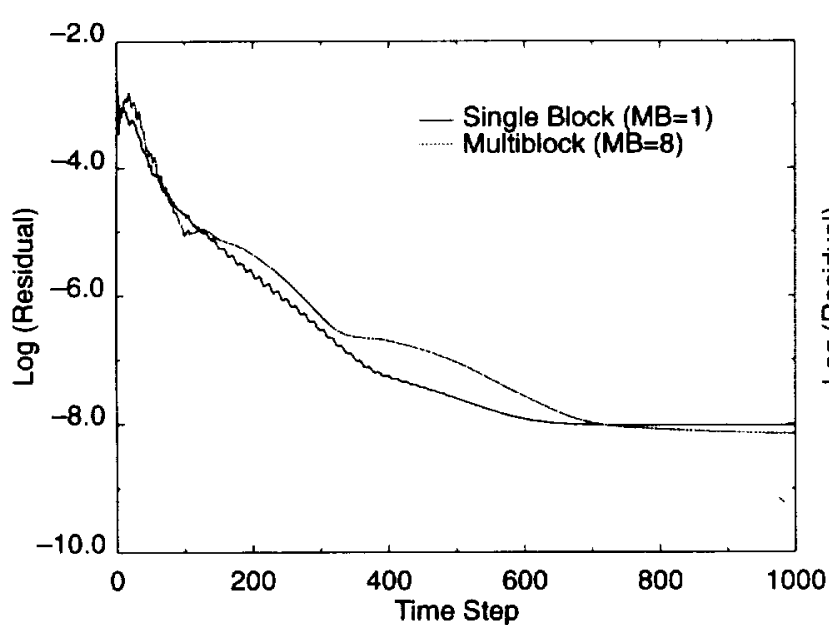

Figure 8. Convergence histories for the 4-element airfoil at $\mathrm{Re}=1 \times 10^{6}$ and $0^{0}$ angle of attack

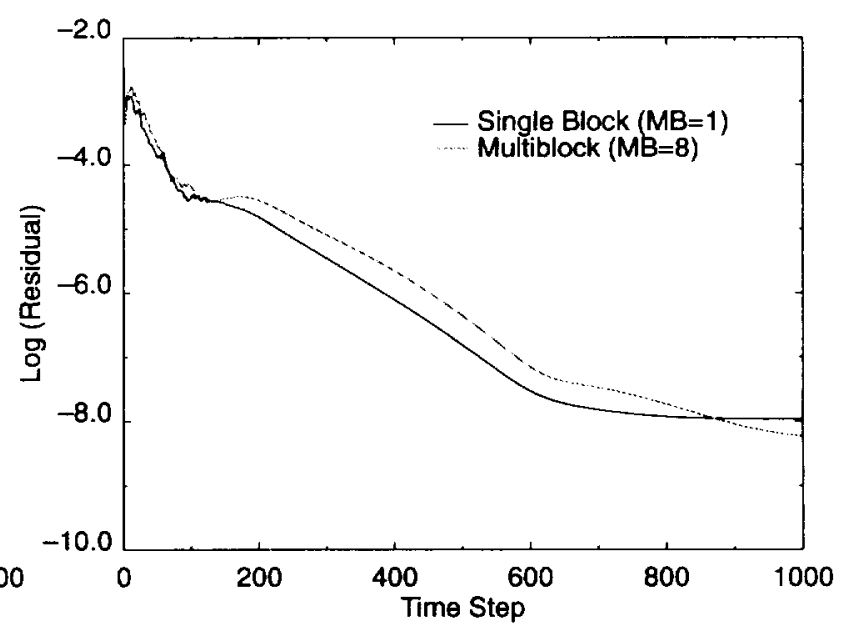

Figure 9. Convergence histories for the 4-element airfoil at $\operatorname{Re}=1 \times 10^{6}$ and $16^{\circ}$ angle of attack 

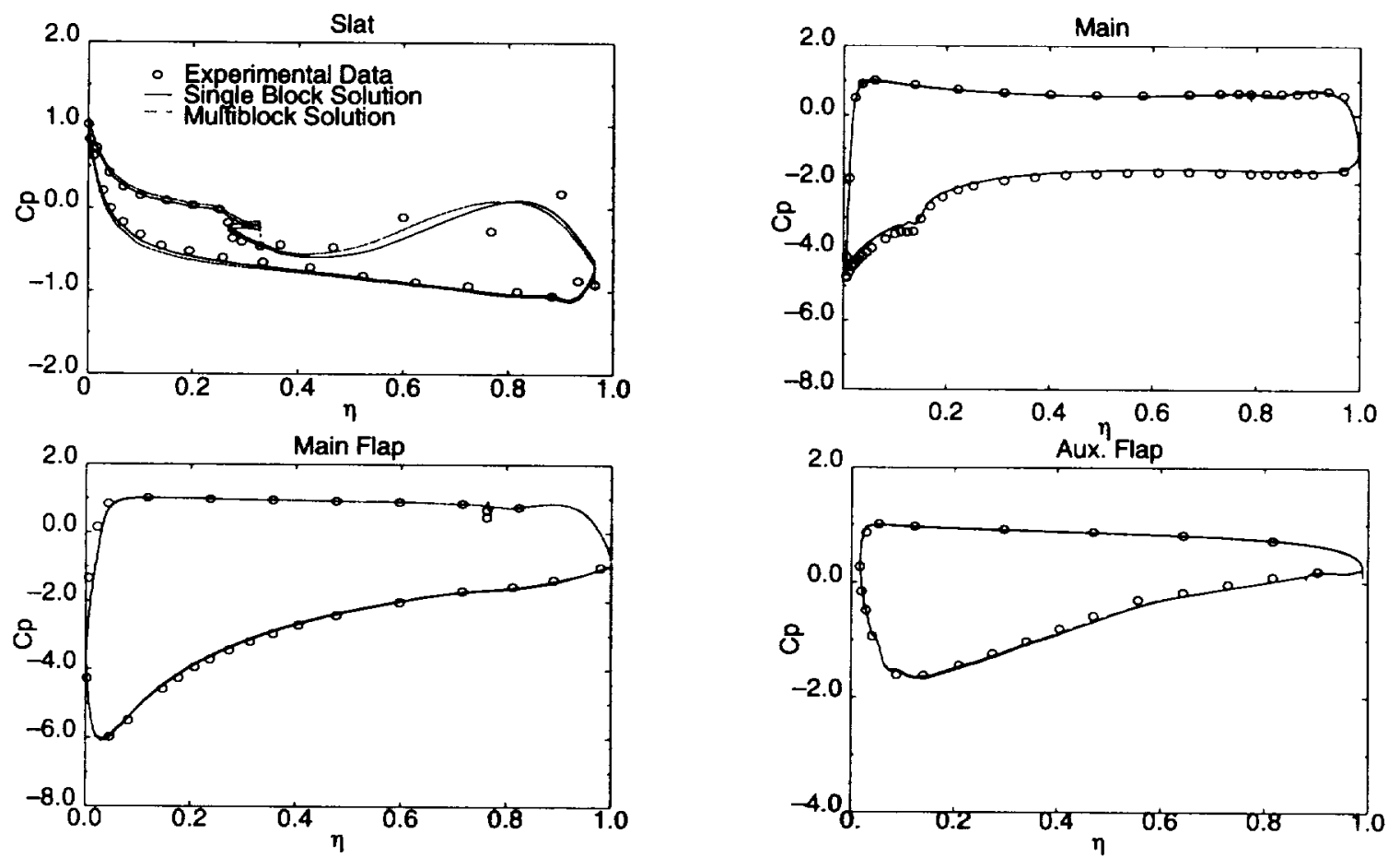

Figure 10. Comparison of $\mathrm{Cp}$ distributions for the 4-element airfoil at $\operatorname{Re}=9 \times 10^{6}$ and $0^{\circ}$ angle of attack
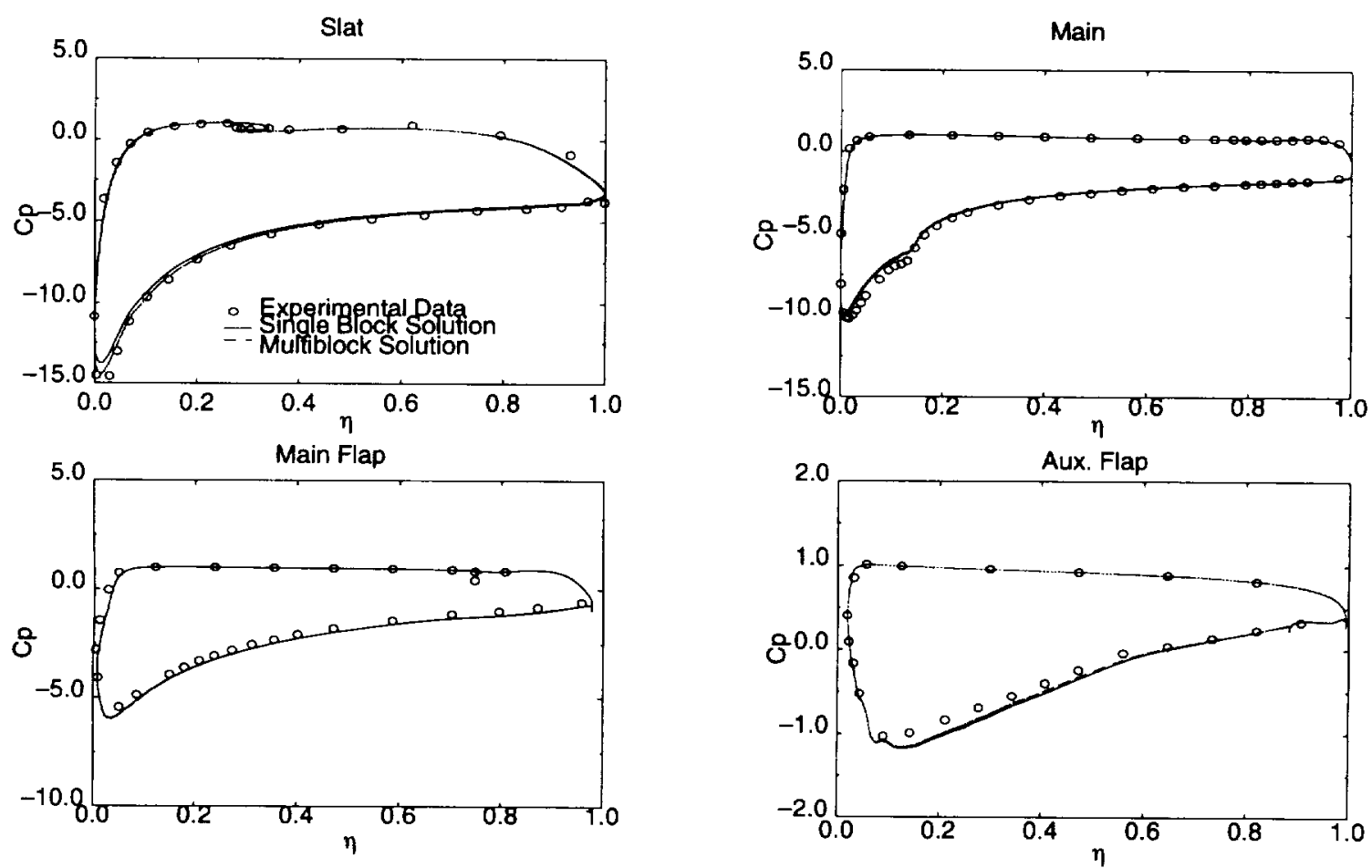

Figure 11. Comparison of $\mathrm{Cp}$ distributions for the 4-element airfoil at $\operatorname{Re}=9 \times 10^{6}$ and $16^{\circ}$ angle of attack 


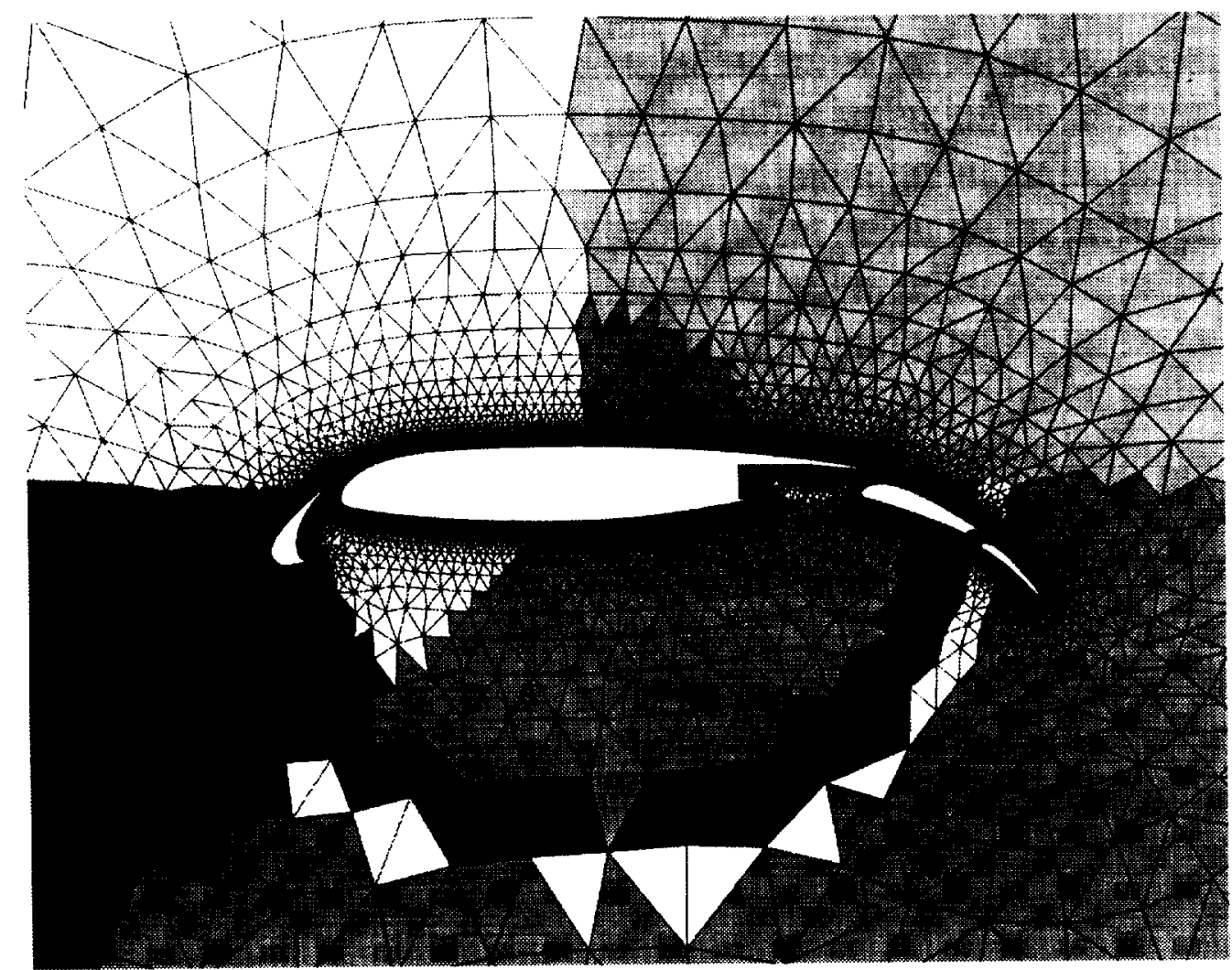

Figure 12. 10-block grid for the 4-element airfoil with 132331 nodes

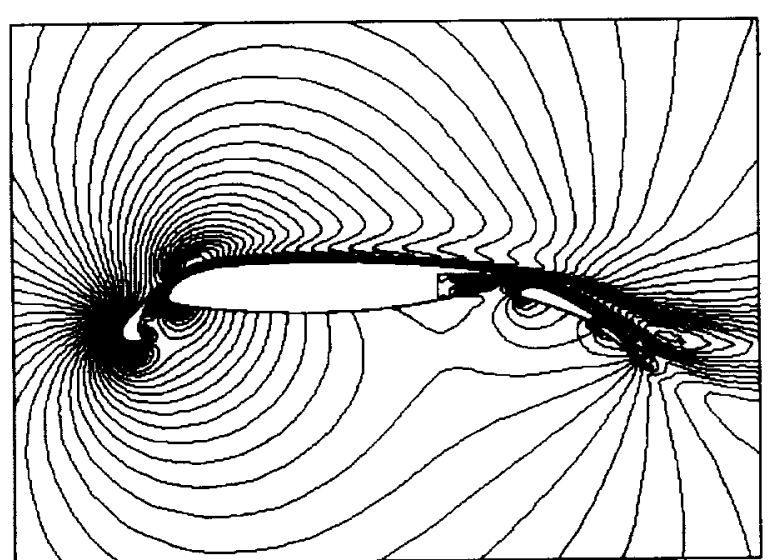

single block

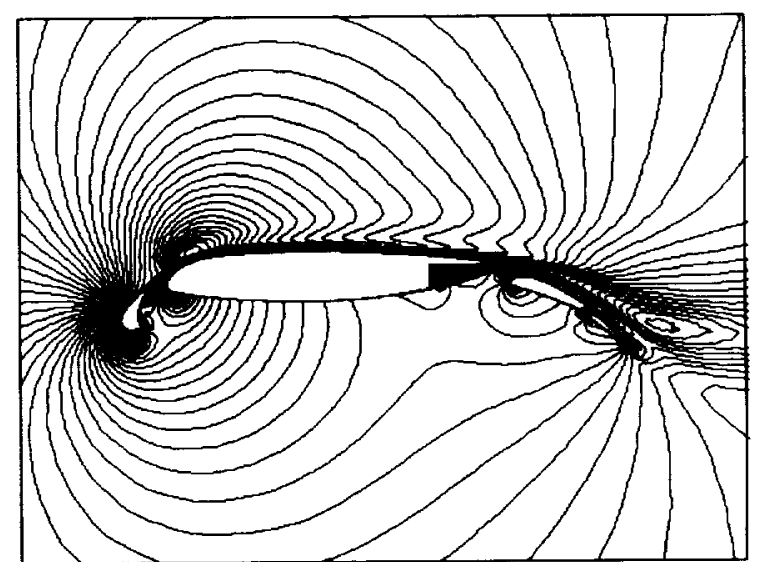

multiblock

Figure 13. Computed u velocity components around the 4-element airfoil on grid with 132331 nodes 


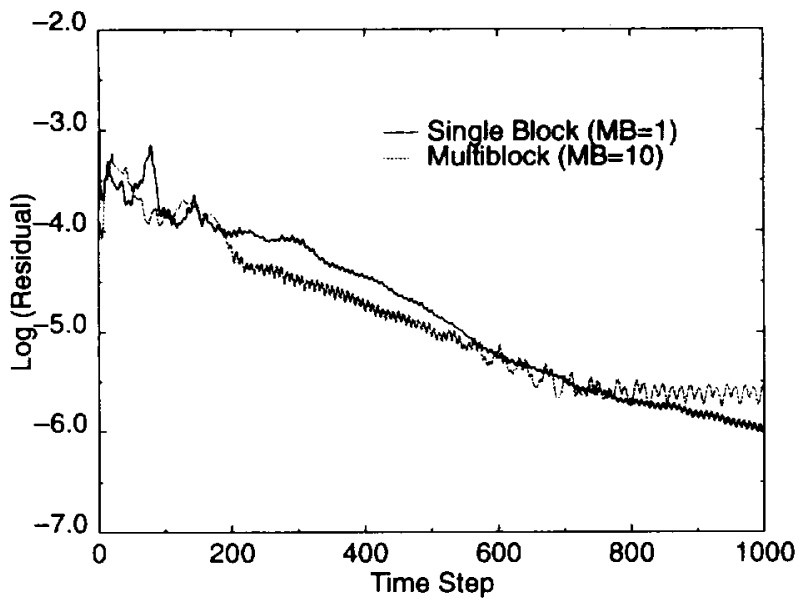

Figure 14. Convergence histories for 4-element airfoil with 132331 nodes

tions. It needs to be pointed that the above single block solutions require about $8-22 \%$ more CPU time than the original code, because of the overhead of the CPU time introduced by recomputing metric quantities of the grid. Even though, the multiblock algorithm still saves about $25 \%$ of the CPU time compared with the original single block algorithm.

\begin{tabular}{|l|c|c|l|}
\hline $\begin{array}{c}\text { Grid } \\
\text { (nodes) }\end{array}$ & Blocks & $\begin{array}{c}\text { Memory } \\
\text { (MB) }\end{array}$ & $\begin{array}{c}\text { CPU Time } \\
\text { (mins) }\end{array}$ \\
\hline 34295 & 1 & 66 & $229(188)$ \\
\hline 34295 & 8 & 25 & 138 \\
\hline 132331 & 1 & 241 & $864(799)$ \\
\hline 132331 & 10 & 69 & 599 \\
\hline
\end{tabular}

Table 2. Memory usage and CPU times of single block and multiblock solutions for 4-element airfoils. The numbers in parenthesis are the CPU time of solutions where the metric terms of the grid are not recomputed.

\section{Conclusions}

The development of a multiblock algorithm to solve the two-dimensional incompressible Reynoldsaveraging Navier-Stokes equations is presented. The multiblock technique has been used in many CFD applications for structured grids. However, little attention has been given to applying this approach to the unstructured grid area. Results presented in this work show that, by properly allocating the memory for the code, the multiblock solution may save up to $70 \%$ of the memory of the single block solution. In fact, over one order of magnitude in memory reduction is possible when the grid is divided into more blocks. Furthermore, the current multiblock algorithm does not introduce any overhead of the CPU time (it even reduces the CPU run time compared with the single block solutions) on the computer used for the current work. However, it is expected that the CPU time may go up some on computers where cache management is less important. Even through, the features demonstrated from the current multiblock algorithm prove that this technique is a useful alternative to the disadvantage associated with the large memory requirements of unstructured grid algorithms. Although the results presented here are for two-dimensional cases, the concepts and methods of the algorithm are readily applicable to general three-dimensional compressible and incompressible flow solvers, and the extension of this approach to three-dimensional flow is underway now. It is believed that the combination of the advantages from the multiblock technology and the unstructured grid technology makes it possible to provide a highly efficient and cost-effective computational tool to predict realistic complex flows about complex configurations.

\section{Acknowledgement}

The authors wish to thank Mr. Gregory Henley of the NSF Engineering Research Center for Computational Field Simulation for helpful discussions.

\section{References}

[1] Anderson, W.K., Rausch, R.D., and Bonhaus, D. L., "Implicit/Multigrid Algorithms for Incompressible Turbulent Flows on Unstructured Grids," AIAA-95-1740, 1995.

[2] Sheng, C., Taylor, L.K., and Whitfield, D.L., "Multiblock Multigrid Solution of Three-Dimensional Incompressible Turbulent Flows About Appended Submarine Configurations," AIAA-95-0203, 33rd Aerospace Sciences Meeting and Exhibit, January 9-12, 1995, Reno, NV.

[3] Sheng, C., Chen, J.P., Taylor, L.K., Jiang, M.Y., and Whitfield, D.L., "Unsteady Multigrid Method For Simulating 3-D Incompressible NavierStokes Flows on Dynamic Relative Motion Grids," AIAA-97-0446, 35th AIAA Aerospace Sciences Meeting and Exhibit, January 6-10, 1997, Reno, NV.

[4] Baldwin, B., and Barth, T., "A One-Equation Turbulence Transport Model for High Reynolds Number Flows of Unstructured Meshes," AIAA-91-0721, 29th Aerospace Sciences Meeting, January, 1991. 
[5] Spalart P., and Allmaras, S., "A One-Equation Turbulence Model for Aerodynamic Flows," AIAA-92-0439, 29th Aerospace Sciences Meeting, January, 1991.

[6] Anderson, W.K., and Bonhaus, D. L., "An Implicit Upwind Algorithm for Computing Turbulent Flows on Unstructured Grids," Computers and Fluids, Vol.23, No.1, 1994, pp.1-21.

[7] Golub, G. and Loan, C.V., Matrix Computations, Johns Hopkins Univ. Press, Baltimore, MD, 1989.

[8] Anderson, W.K., "Grid Generation and Flow Solution Method For Euler Equations on Unstructured Grids," NASA Technical Report TM4295, 1992.
[9] Batina, J.T., "Implicit Flux-Split Euler Schemes for Unsteady Aerodynamic Analysis Involving Unstructured Dynamic Meshes," AIAA-900936, 1990.

[10] Barth, T.J., and Linton, S.W., "An Unstructured Mesh Newton Solver for Compressible Fluid Flow and Its Parallel Implementation," AIAA-95-0221, 33rd Aerospace Sciences Meeting, January 9-12, 1995, Reno, NV.

[11] Coles, D. and Wadcock, A.J., "Flying-HotWire Study of Flow Past an NACA 4412 Airfoil at Maximum Lift," AIAA Journal, Vol. 17, No. 4, April 1979.

[12] Valarezo, W., Dominik, C., McGhee, R., Goodman, W., and Paschal, K., "Multi-Element Airfoil Optimization for Maximum Lift at High Reynolds Number," AIAA-91-3332-CP, 1991. 